\title{
Efecto del dragado de un canal de comunicación al mar (estero) sobre la asociación de peces en la Laguna Larga, Cayo Coco, Cuba
}

\author{
Effect of the dredging of an estuarine channel connecting to the \\ sea on fish distribution in Laguna Larga, Cayo Coco, Cuba
}

\author{
Lisadys Bárbara Dulce Sotolongo ${ }^{1 *}$ y Fabián Pina Amargós ${ }^{1}$
}

\begin{abstract}
RESUMEN
En este trabajo se caracterizaron el hábitat y la comunidad de peces en el canal de comunicación al mar de Laguna Larga, antes y después de un proceso de dragado realizado en el año 2012. Para ello el canal se dividió en cuatro secciones, dos iniciales cercanas al mar y dos finales cercanas a la laguna. Se realizaron 112 censos visuales mediante buceo libre. Para caracterizar el hábitat se midieron el área de refugio, sombra, temperatura del agua, salinidad, profundidad y velocidad de la corriente. Se determinaron la distribución espacio-temporal de los peces, riqueza de especies, densidad y frecuencia relativa en cada sección y se relacionaron con las características del hábitat. Se registraron 22 especies de peces distribuidas en 14 familias. Las especies Abudefduf saxatilis, Halichoeres bivittatus, Stegastes leucostictus y Lutjanus griseus fueron dominantes. Se encontró que las densidades y riquezas de peces en el inicio del canal eran más altas que en el extremo, particularmente la especie Lutjanus griseus, la cual cambió sus preferencias de nichos, desde el final del canal hasta el inicio. Se encontró que las comunidades de peces variaron espacial y temporalmente. El proceso de restauración produjo una disminución en la disponibilidad de refugios y, en consecuencia, determinó la disminución de la densidad y riqueza de especies solo en el canal de entrada a la laguna. Los cambios más drásticos en el patrón espacial y temporal de los peces estuvieron asociados a las secciones finales del canal, las cuales sufrieron las mayores alteraciones de hábitat.
\end{abstract}

Palabras claves: Comunidad de peces, canal de comunicación, Laguna Larga, efecto, restauración.

\begin{abstract}
Fish community and habitat of the estuarine channel connecting to the sea at Laguna Larga were characterized before and after the dredging conducted in 2012. For this purpose, the channel was divided into four sections: two initial sections near the sea (IA, IB) and two final sections near the lagoon (FA, FB). A total of 112 visual surveys were conducted by free diving. Shelter area, shade, water temperature, salinity, as well as current depth and velocity were measured in order to characterize the habitat. Spatial and temporal distribution of fish, species richness, density, and relative frequency were determined in each section, and results were related to habitat characteristics. A total of 22 species of fish distributed in 14 families were recorded. The following were the dominant species: Abudefduf saxatilis, Halichoeres bivittatus, Stegastes leucostictus, and Lutjanus griseus. Fish density and richness were higher at the beginning of the channel than at the end, particularly Lutjanus griseus, which changed its niche preferences from the end of the channel (FB) to the beginning (IB). In addition, fish communities varied spatially and temporally. The restoration process resulted in decreased availability of shelter and consequently determined the decrease in density and species richness at the beginning of the channel. The most drastic changes in fish spatial and temporal pattern were associated with the final sections of the canal, which suffered the greatest changes in habitat.
\end{abstract}

Keywords: Fish community, estuarine channel, Laguna Larga, effect, restoration.

Centro de Investigaciones de Ecosistemas Costeros (CIEC). Cayo Coco. Morón. CP: 69400. Ciego de Ávila. Cuba. lisy.delfin@gmail.com*, fabianpina1972@gmail.com

Recibido: 19 de marzo de 2017

Corregido: 23 de octubre de 2017

Aceptado: 24 de octubre de 2017

DOI: http://dx.doi.org/10.15359/revmar.9-2.2 


\section{INTRODUCCIÓN}

En Cuba, dada su condición de insularidad, el ecosistema de manglar tiene una gran importancia económica, ecológica y estratégica, debido a la franja boscosa que ocupa en las costas; es utilizado como refugio para muchas especies, tanto en sus estados larvales como juveniles, mantiene la línea costera y evita la erosión de las costas (estabilización de los sedimentos). La fauna que habita en los manglares controla el detritus y facilita la degradación por parte de los microorganismos, además ocupa cerca del $5 \%$ de la superficie del país y del $26 \%$ de la cobertura boscosa (Menéndez \& Priego, 1994). Con frecuencia, a estos hábitats se les otorga la condición de sitios de crianza porque, aparentemente, hay un incremento de la supervivencia de juveniles (Nagelkerken et al. 2000; 2001).

El manglar es un factor dominante que estructura las comunidades de peces de arrecife, no solo explica un componente significativo de la estructura de la comunidad, sino que por lo general influye en los sistemas arrecifales (Nagelkerken et al. 2002). Algunos autores sugieren que los manglares proporcionan una etapa de hábitat intermedia entre los lechos de pastos marinos y los arrecifes (Nagelkerken et al. 2002). Además, mejoran la biomasa de los peces en los arrecifes del Caribe, porque los ecosistemas costeros tropicales están funcionalmente vinculados (Nagelkerken et al. 2002). Dada la cada vez mayor variedad y gravedad de las perturbaciones naturales y antropogénicas de los arrecifes de coral (Knowlton, 2001), se debe conservar cualquier fuente natural de producción y resiliencia de los ecosistemas. La actual tasa de deforestación del manglar tendrá consecuencias nocivas significativas para el funcionamiento, las pesquerías, la biodiversidad y la resiliencia de los arrecifes de coral del Caribe (Nagelkerken et al. 2002).

Recientemente se ha encontrado que la ictiofauna de barreras de coral depende de la presencia de manglares. La biomasa de las poblaciones de importancia pesquera es más del doble cuando hay presencia de manglares (Mumby et al. 2004).

Las lagunas costeras son áreas utilizadas comúnmente para la protección, alimentación y reproducción de muchos organismos marinos, por lo que gran número de especies dependen de la conservación de estos ecosistemas. Sus características hidrológicas y ecológicas hacen de ellas áreas con hábitats que manifies$\tan$ variaciones estacionales significativas. Esto reviste una gran importancia desde la perspectiva de la investigación científica y de la conservación de la biodiversidad. Por pequeñas que sean, mantienen una vida particular en su interior $y$, generalmente, son sitios donde la biodiversidad asociada es un atributo muy importante (Contreras \& Castañeda, 2004).

Los canales de comunicación asociados a ellas se caracterizan por tener fuertes gradientes direccionales de salinidad, materia orgánica, nutrientes y concentración de oxígeno; los cuales actúan 
como filtro en la colonización de especies (Garrido et al. 2011). Por lo cual las modificaciones en las comunidades de peces pueden ser el resultado de cambios ambientales (Garrido et al. 2011; Farré et al. 2015; Sirot et al. 2015). Aunque la baja segregación espacial de peces en lo referente a preferencia alimenticia indica que, en una laguna y su canal adyacente con amplia disponibilidad de alimentos, los factores abióticos parecen tener un mayor efecto en la distribución de los peces que las interacciones biológicas (Pombo et al. 2005). Farré et al. (2015) plantean que, a escalas pequeñas, las variables que modifican la estructura de las comunidades de peces son: la profundidad, el tipo de fondo, la complejidad del hábitat y los aportes terrestres. La diversidad de la comunidad de peces disminuye por un empobrecimiento de la vegetación del fondo, dado que hábitats con pastos, generalmente, soportan una gran variedad de fauna (Villéger et al. 2010; MohamedHarris \& Vinobaba, 2013). Por ende, una alta complejidad del hábitat provee mayor cantidad de alimentos y refugio contra depredadores (Caballero-Vázquez \& Vega-Cendejas, 2012). No puede obviarse que cada especie percibe el ambiente en un rango de escala único y esto responde a la variabilidad individual (Mesa et al. 2010). Sin embargo, las características del sedimento, la heterogeneidad del sustrato y la cobertura vegetal también afectan la distribución de peces, dada su influencia en la disponibilidad de presas (Mesa et al. 2010) y la protección contra depredadores (Pombo et al. 2005). La eutrofización, como resultado de la acción del hombre sobre el ecosistema, induce cambios ma- yores en la estructura de las comunidades de peces (Benedetti-Cecchi et al. 2001). También puede modificar la composición de especies del fitoplancton produciendo anomalías que tienen importantes repercusiones en la composición de la ictiofauna (Nixon \& Fulweiler, 2009).

Laguna Larga es una laguna costera cuya única vía de comunicación con el mar es un canal de $500 \mathrm{~m}$ de largo y un ancho promedio de $8 \mathrm{~m}$. Es una de las lagunas que posee evidencias de ser, no solo un sitio de refugio para especies de peces, sino también un posible sitio de cría y reproducción (Guimarais-Bermejo, 2007; Hernández-Roque, 2007; Olivera-Espinosa, 2013; Salvat-Torres et al. 2013). Estudios realizados por SalvatTorres et al. (2010; 2013) demostraron que la comunidad de peces en el canal presenta una marcada segregación espacial, relacionada con las características del hábitat (disponibilidad de refugio y sombra) y la distancia de las zonas del canal a la zona marina aledaña; también se mostraron variaciones en el tiempo, al parecer vinculadas con la movilidad intrínseca de los peces, las migraciones reproductivas, el reclutamiento y la mortalidad diferencial asociada a la depredación. Además, presentan diferentes grados de movilidad, pero utilizan el manglar como zona de refugio y con poca actividad de forrajeo. Se han realizado otras investigaciones, relacionadas con la caracterización fisicoquímica de sus aguas, pastos marinos, productividad primaria del fitoplanton (Guimarais-Bermejo, 2007; Hernández-Roque, 2007), y con la fauna de moluscos marinos (Olivera-Espinosa, 2013). 
Laguna Larga presenta un alto grado de antropización a causa de la construcción de cuatro hoteles asentados en sus márgenes (sobre todo en la parte sur). Zúñiga \& González (2000) plantean que acciones como el desbroce de vegetación (fundamentalmente del mangle), el relleno y compactación de áreas de la laguna, así como el vertimiento de residuales líquidos y sólidos, son las causas principales de la reducción del intercambio de agua de esta laguna con el mar a través de su canal de comunicación en los últimos 12 años. En el año 2011 se puso en marcha un proyecto de restauración en dicha laguna con vista a mejorar el intercambio de agua con el mar. Las primeras acciones han sido trabajos de dragado entre los lóbulos de esta laguna, los cuales deberían mejorar el flujo de agua. Este proceso produjo un incremento en la calidad del agua y en la fuerza de la corriente de marea que sale por el canal de comunicación al mar y, por ende, cambiaron las características del hábitat y el comportamiento de algunas variables abióticas y con ellos provocaron modificaciones en la biota asociada. Debido a la afirmación anterior podría ser que el proceso de dragado realizado en Laguna Larga, aumentara la densidad de peces en su canal de comunicación al mar. Este trabajo se propone caracterizar y comparar el hábitat y la comunidad de peces del canal de comunicación al mar, antes y después del proceso de dragado de Laguna Larga.

\section{MATERIALES Y MÉTODOS Área de estudio}

La investigación se realizó en el canal de comunicación al mar de Laguna Larga, ubicada en la costa norte de Cayo Coco (grupo insular Jardines del Rey), Cuba, en los $22^{\circ} 32^{\prime}$ $14.59^{\prime}$ ' N y los $78^{\circ} 21^{\prime} 25.33^{\prime}$ ' W (Fig. 1). Laguna Larga es un cuerpo de agua alargado y estrecho, con una longitud de $2912 \mathrm{~m}$ y un ancho promedio de $79 \mathrm{~m}$, con una superficie de $2.3 \mathrm{~km}^{2}$ y posee entre $3 \mathrm{~m} \mathrm{y} 4 \mathrm{~m}$ en su parte más estrecha, hasta $186 \mathrm{~m}$ en su parte más ancha. El intervalo de marea en la región es semidiurna regular y con una amplitud relativamente pequeña (González-De Zayas, 2012).

La batimetría es uniforme y se caracteriza por poseer un fondo de sedimentos fangosos, aunque en algunas porciones presenta substratos rocosos, con restos de materiales de construcción, originados por el desarrollo hotelero y la arena. La evolución natural de esta laguna litoral está relacionada con la autocolmatación, debido al crecimiento acentuado de $R$. mangle, mejor conocido como mangle rojo, en la salida hacia el mar. Como consecuencia, se ha observado una disminución paulatina de los niveles de intercambio de agua hacia el resto de la laguna. Por otra parte, han ocurrido modificaciones asociadas al desarrollo hotelero (dragados, rellenos y cierres temporales), las cuales han generado condiciones hidrodinámicas que permiten analizar cada sector por separado como ecosistemas lagunares diferentes.

El canal está bordeado por mangle rojo y en menor proporción por $A$. 


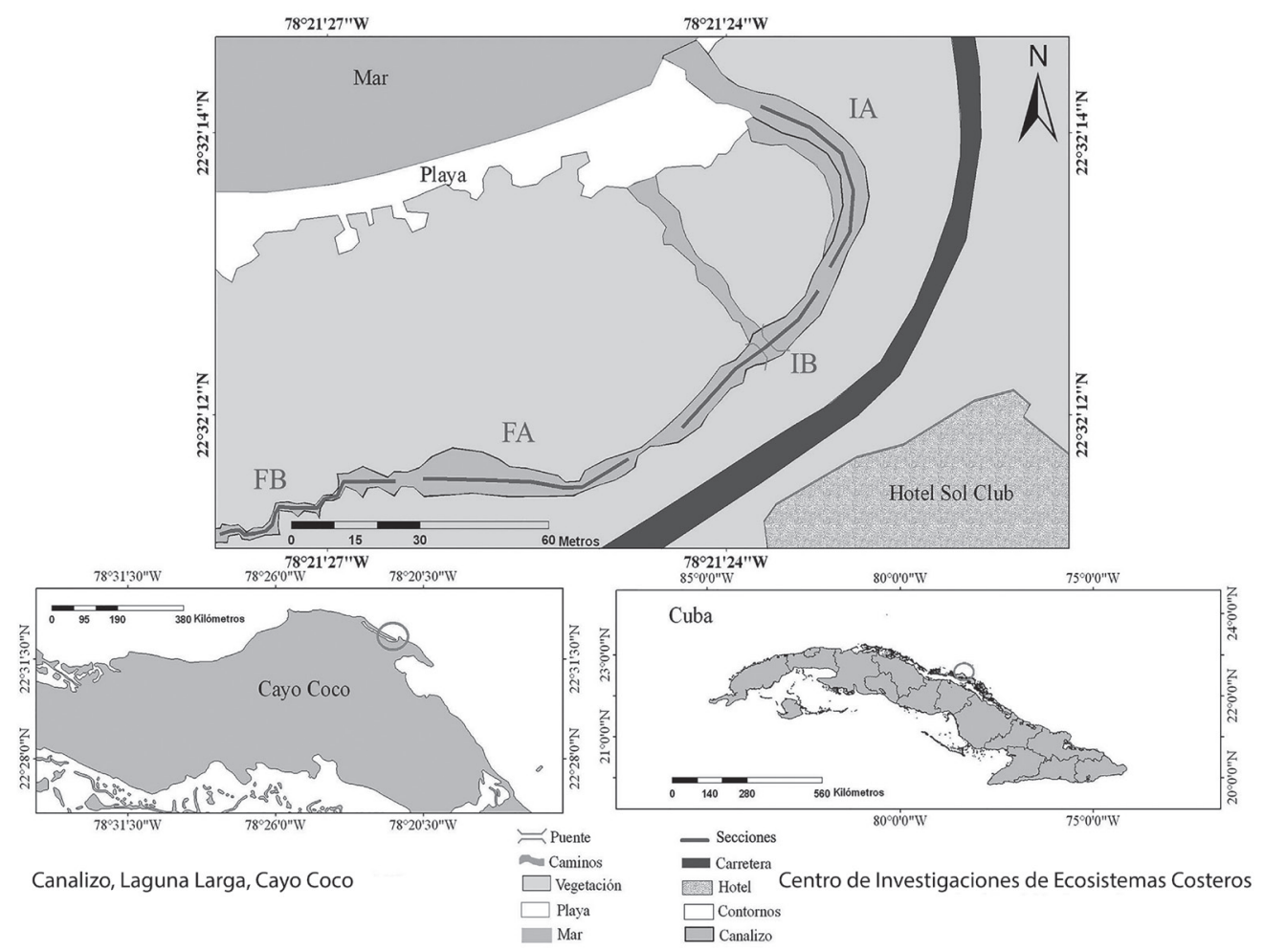

Fig. 1. Ubicación del área de estudio en la Laguna Larga, Cayo Coco, Cuba

Fig. 1. Location of study area in Laguna Larga, Cayo Coco, Cuba

germinans, mejor conocido como mangle prieto (Salvat-Torres et al. 2010).

\section{Diseño y método de muestreo}

El canal fue dividido en cuatro secciones (IA, IB, FA y FB) (Fig. 1), dos iniciales más cercanas al mar (IAIB) y dos finales más hacia el interior de la laguna (FA-FB). Ambas zonas están separadas por una distancia de $30 \mathrm{~m}$. Cada una de las secciones constituyó un recorrido de $50 \mathrm{~m}$ de longitud y $2 \mathrm{~m}$ de ancho en buceo libre (Brock, 1954; Rogers et al. 1994), se marcó el inicio y el final de cada sección con cintas de colores para establecer estaciones fijas de muestreo, siguiendo los mismos criterios que la metodología utilizada por Salvat-Torres et al. $(2010 ; 2013)$ para la obtención de los datos predragado. Las evaluaciones en el canal se efectuaron desde marzo del 2014 hasta febrero del 2015, con una frecuencia de tres veces al mes. Se utilizaron los datos de 776 censos realizados pertenecientes a los años 2007 y 2008 antes del mismo proceso (Salvat-Torres et al. 2010; 2013) para establecer la comparación de densidad y riqueza de especies luego del dragado realizado en el año 2012.

Fueron realizados un total de 112 censos visuales, utilizando una variante del método de censo visual 
(Brock, 1954; Rogers et al. 1994), en buceo libre. Se cuantificaron las especies, el número de individuos y la talla estimada de los peces observados desde la ribera del canal hacia el centro, en un ancho de $2 \mathrm{~m}$. Con estos datos se calcularon la riqueza de especies y la densidad de cada individuo (Locatelli \& Petersen, 1975; StatSoft, 2011).

En cada muestreo se anotaron el estado de la marea, la fase lunar y la hora de realización. La clasificación sistemática utilizada para la confección de la lista de especies de los peces siguió los criterios de Claro \& Parenti (2001). Las especies Scarus iserti y Scarus taenopterus se trataron como un complejo porque los juveniles no se pueden diferenciar visualmente (González-Sansón \& Aguilar, 2003).

\section{Características del hábitat}

Para caracterizar el hábitat, se midió el área de sombra y refugio en los bordes, en el centro y en el fondo del canal, utilizando una cinta métrica. En los bordes se identificaron tres tipos de refugio: las raíces (zanco) de mangle rojo, las raíces (neumatóforos) de mangle prieto y la cobertura vegetal que formaba sombra sobre la superficie del agua. En el centro se midieron linealmente, la proporción de arena de Thalassia testudinum Banks ex König, algas, piedras, troncos y depósitos de materia vegetal en descomposición usando una cinta métrica. El fondo, en el centro del canal, se caracterizó según el método de transecto intercecto (Rogers et al. 1994), en cada metro de las secciones definidas, la utilización de esta metodología siguió los mismos criterios que la empleada por SalvatTorres et al. $(2010 ; 2013)$ para la obtención de los datos predragado en cuanto a las características del hábitat.

\section{Parámetros fisicoquímicos}

Los parámetros fisicoquímicos medidos fueron: profundidad máxima del canal, temperatura, salinidad, oxígeno disuelto, $\mathrm{pH}$ del agua y la velocidad media de la corriente de marea. Estos se midieron en cada sección en el primer y cuarto días del cambio de fase lunar, a la hora de la realización de los censos y en el punto medio de la marea, para evitar los momentos extremos del ciclo mareal en los cuales cambian muy rápido el sentido y la intensidad de la corriente. La profundidad fue medida en tres puntos de cada sección, con una vara graduada de 5 $\mathrm{cm}$ de precisión. La temperatura fue medida con un termómetro de precisión $0.1^{\circ} \mathrm{C}$ y a $10 \mathrm{~cm}$ de la superficie, al inicio y al final de cada sección, al igual que la salinidad, el oxígeno disuelto y el $\mathrm{pH}$. La salinidad fue medida con un refractómetro-salinómetro marca ATAGO de 1 UPS de precisión, con agua de la superficie. Se tomó una muestra de agua en un frasco plástico y se midieron el $\mathrm{pH}$ y el oxígeno disuelto en agua en el laboratorio de química utilizando un oxímetro de marca HACH sension 8 (modelo 50180) y un phmetro de marca HANNA (modelo pH 213), respectivamente. Para determinar la velocidad media de la corriente de marea, se midió cinco veces el tiempo en que una boya, a media 
agua, recorría una distancia a $10 \mathrm{~cm}$ de profundidad, en dos puntos de cada sección; a partir de estas mediciones se calculó la velocidad.

\section{Procesamiento de datos}

Para el análisis de los datos se calculó la densidad media (individuos $/ 100 \mathrm{~m}^{2}$ ). La densidad de peces antes y después del proceso de dragado fue comparada estadísticamente utilizando las pruebas no paramétricas de Friedman (Zar, 1996). No se utilizó Análisis de varianza (ANOVA), por no cumplirse las asunciones necesarias para su adecuada interpretación. Los tamaños de muestra para las diferentes variables fueron suficientes para la estimación de los parámetros descriptivos con un error estándar menor al 10\% (Locatelli \& Petersen, 1975). Los análisis se hicieron en el programa estadístico STATISTICA versión 10.0 (StatSoft, 2011).

La densidad de las familias Clupleidae y Atherinidae se analizó en forma independiente debido a que estas incluyen especies de tamaños muy pequeños y con frecuencia relativamente baja, pero que conforman cardúmenes de muchos ejemplares, lo cual puede sesgar los resultados de densidad total y confundir las tendencias.

Se determinó la riqueza de Margalef con el empleo del programa PRIMER, versión 6.0 (Clarke \& Gorley, 2006) y se comparó entre años a través de la Prueba de Friedman.

Los datos de cobertura de mangle rojo, mangle prieto, sombra, cobertura de arena, profundidad y variables fisicoquímicas, antes y después del proceso de dragado, fueron comparados utilizando la prueba no paramétrica de Mann-Whitney (Zar, 1996), cuya cobertura fue determinada como: la suma de la cantidad de raíces de mangle rojo y mangle prieto presentes en las riberas del canal entre el área $\left(100 \mathrm{~m}^{2}\right)$, al igual fue cuantificada la cobertura de sombra.

\section{RESULTADOS \\ Densidad de especies}

Las familias más destacadas fueron tres, cinco menos que las reportadas por Salvat-Torres et al. (2010; 2013), coincidiendo dos: Pomacentridae y Labridae. Las familias Clupleidae y Atherinidae siguieron siendo las familias con mayor densidad media total por la contribución de las sardinas y las manjúas. Aunque estas densidades no se mantuvieron todo el tiempo, sino que oscilaron sustancialmente.

La composición por tallas de los peces en el canal muestra que la mayoría de las especies son juveniles, con predominio de los peces de tallas pequeñas menores de $5 \mathrm{~cm}$. Se observan muy pocos ejemplares de tallas mayores de $20 \mathrm{~cm}$. Las familias Haemulidae y Lutjanidae presentaron mayor proporción de individuos en el grupo de tallas de 20.1-30 cm, mientras que Gerridae, Labridae, Pomacentridae y Scaridae fueron más abundantes en las tallas pequeñas $(\leq 5 \mathrm{~cm})$.

El análisis de la densidad media de peces en el canal en los años de muestreos 2014-2015 indicó que esta fue similar a los años 2007 y 2008, pero significativamente menor, disminuyendo continuamente de 
manera significativa desde el año 2014 al $2015(P<0.03$, ANOVA de Friedman), según datos utilizados en estudios realizados por Salvat-Torres et al. $(2010 ; 2013)$ antes del dragado, empleándose a su vez el mismo procedimiento para ser cuantificadas y comparadas ambas densidades (Fig. 2).

\section{Distribución espacio-temporal de las especies dominantes}

Los resultados obtenidos antes del proceso de dragado muestran una marcada segregación espacial de la abundancia y el tamaño de las especies en la comunidad de peces presentes en el canal. Se observó que el patrón general de distribución de la ictiofauna se mantiene igual en los dos años de estudio, y que la abundancia media de la mayoría de los peces disminuía hacia el final del canal (Salvat-Torres et al. 2010; 2013).

El seguimiento en el tiempo de la distribución espacial de la comunidad de peces del canal demuestra que su estructura no cambia con el tiempo, sino las abundancias de sus poblaciones individualmente (SalvatTorres et al. 2010; 2013).

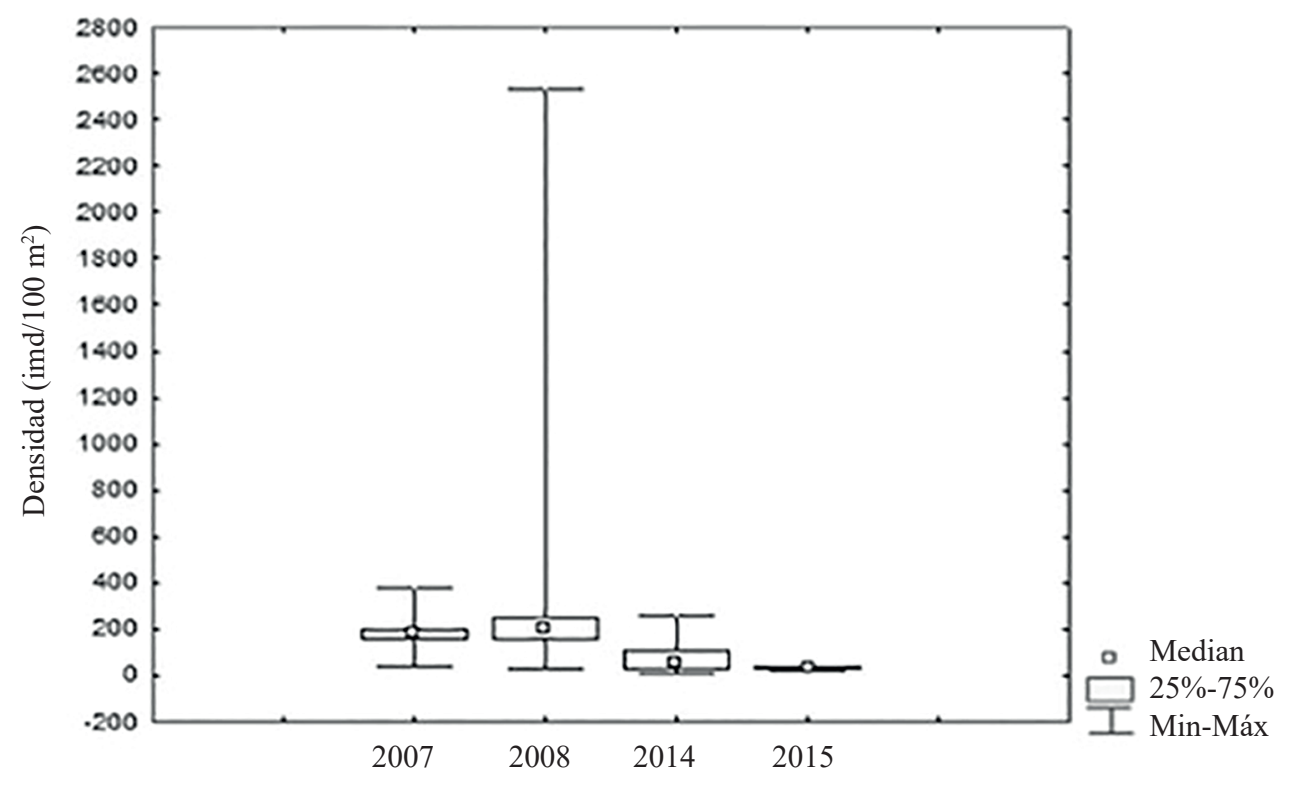

Años

Fig. 2. Densidad media total de los peces en el canal de comunicación al mar de Laguna Larga entre los años 2007 y 2015, sin incluir las familias Clupleidae-Atherinidae. (Diferencias significativas, $P<0.03$, ANOVA de Friedman)

Fig. 2. Total average fish density in the Laguna Larga estuarine channel between 2007 and 2015, except for the Clupleidae-Atherinidae families. (Significant differences, $P<0.03$, Friedman's ANOVA) 

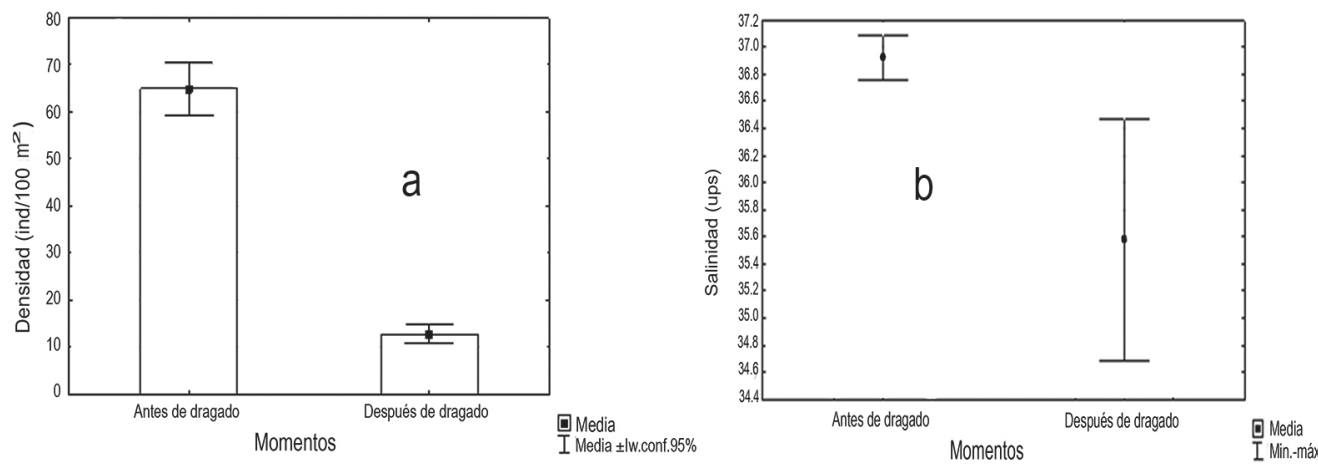

Fig. 3a. Densidad media de las 4 especies dominantes antes y después del proceso de dragado, $(P<0.001$, Prueba U de Mann-Whitney). b. Salinidad del agua en el canal de comunicación al mar de Laguna Larga antes y después del proceso de dragado. (Diferencias significativas, $P<0.01$, Prueba U de Mann-Whitney)

Fig. 3a. Average density of the 4 dominant species, before and after the dredging process, $(P<0.001$, Mann-Whitney U Test $)$, b. Water salinity in the Laguna Larga estuarine channel before and after the dredging process. (Significant differences, $P<0.01$, MannWhitney U Test)

La densidad media de las cuatro especies dominantes (Lutjanus griseus L., Abudefduf saxatilis L., Halichoeres bivittatus Bloch.y Stegastes leucostictus Müller \& Troschel) en el canal de comunicación al mar de Laguna Larga mostró patrones variados.

Se pudo comprobar que las poblaciones de $A$. saxatilis, $H$. bivittatus y $S$. leucostictus, se concentraban en las dos primeras secciones de los canales IA e IB, mientras que L. griseus mostró una distribución alternante, con un máximo de densidad en el IB y un mínimo en el FA (Fig. 3).

A. saxatilis se mantuvo como la especie dominante durante todos los muestreos, seguida por $H$. bivittatus, $S$. leucostictus, L. griseus, los juveniles de Scarus iserti-taenopterus Dermarest., especies de la familia Eusinostomidae y Haemulon parra Dermarest. Las especies con mayor frecuencia relativa fueron: L. griseus, Gerres cinereus, A. saxatilis, H. bivittatus, Eucinostomus sp., S. leucostictus, H. sciurus, Sphyraena barracuda y L. apodus (Cuadro 1).

La densidad media de las cuatro especies dominantes en el canal (antes de los años 2007 y 2008, y después del proceso de dragado en los años 2014 y 2015) que sufrió Laguna Larga también se redujo. El número de individuos presentes en el canal disminuyó sustancialmente después de que se realizó el dragado en la laguna $(P<0.001$, Prueba U de MannWhitney) (Fig. 3a).

\section{Características del hábitat}

El canal no era homogéneo en cuanto a las características de sus riberas antes del proceso de dragado, sus bordes tenían más cobertura de raíces y sombra de mangle rojo hacia el final del canal, mientras que en su 
Cuadro 1. Densidad media total (individuos $/ 100 \mathrm{~m}^{2}$ ) y frecuencia de observación, en los censos de las especies más conspicuas después del dragado en el canal de comunicación al mar de Laguna Larga

Table 1. Total average density (individuals $/ 100 \mathrm{~m}^{2}$ ) and frequency of observation in the surveys of the most conspicuous species after the dredging in the estuarine channel of Laguna Larga

\begin{tabular}{lccccc}
\hline Especies & Media & Máximo & $\begin{array}{c}\text { Desviac. } \\
\text { estánd. }\end{array}$ & $\begin{array}{c}\text { Error } \\
\text { estánd. }\end{array}$ & $\begin{array}{c}\text { Frecuencia de } \\
\text { observación }\end{array}$ \\
\hline Clupleidae-Atherinidae & 73.22 & 5000.00 & 402.08 & 12.72 & 10 \\
Abudefduf saxatilis & 53.24 & 372.00 & 75.45 & 2.39 & 87 \\
Halichoeres bivittatus & 11.03 & 203.00 & 15.61 & 0.49 & 82 \\
Stegastes leucostictus & 9.54 & 187.00 & 13.57 & 0.43 & 78 \\
Lutjanus griseus & 7.92 & 85.00 & 10.13 & 0.32 & 95 \\
Scarus iserti-taenopterus & 6.48 & 106.00 & 11.75 & 0.37 & 55 \\
Eusinostomus sp. & 5.60 & 60.00 & 8.39 & 0.27 & 82 \\
Haemulon parra & 4.50 & 27.00 & 5.51 & 0.17 & 61 \\
Gerres cinereus & 3.86 & 26.00 & 3.99 & 0.13 & 94 \\
Lutjanus apodus & 2.56 & 40.00 & 3.59 & 0.11 & 70 \\
Sparisoma aurofrenatum & 2.15 & 45.00 & 3.73 & 0.12 & 77 \\
Haemulon sciurus & 2.10 & 24.00 & 2.89 & 0.09 & 59 \\
Chaetodon capistratus & 1.12 & 11.00 & 1.52 & 0.05 & 77 \\
Strongylura notata & 1.11 & 30.00 & 3.17 & 0.10 & 44 \\
Sphoeroides testudineus & 0.95 & 14.00 & 1.72 & 0.05 & 49 \\
Stegastes fuscus & 0.81 & 11.00 & 1.42 & 0.04 & 14 \\
Sphyraena barracuda & 0.57 & 5.00 & 0.83 & 0.03 & 48 \\
Canthigaster rostrata & 0.20 & 12.00 & 0.69 & 0.02 & 32 \\
Acanthurus chirurgus & 0.16 & 5.00 & 0.56 & 0.02 & 23 \\
Lutjanus cyanopterus & 0.06 & 4.00 & 0.28 & 0.01 & 14 \\
Pterois volitans & 0.02 & 2.00 & 0.17 & 0.01 & 33 \\
Haemulon flavolineatum & 0.01 & 2.00 & 0.08 & 0.00 & 27 \\
\hline & & & & & \\
\hline
\end{tabular}

inicio, predominaban los neumatóforos y raíces comunes de mangle prieto. Las riberas del canal no son homogéneas, pues sus bordes tienen más cobertura de raíces y sombra de mangle rojo (Rhizophora mangle) hacia el final del canal que hacia el inicio, donde hay predominio de neumatóforos y raíces de mangle prieto (Avicennia germinans). Con respecto al fondo del canal, el predominio de algas macroscópicas es mayor en el inicio del canal y los hidrozoos y Thalassia predominan hacia el final de este.

Luego del proceso de dragado esta composición tendió a homogeneizarse, 


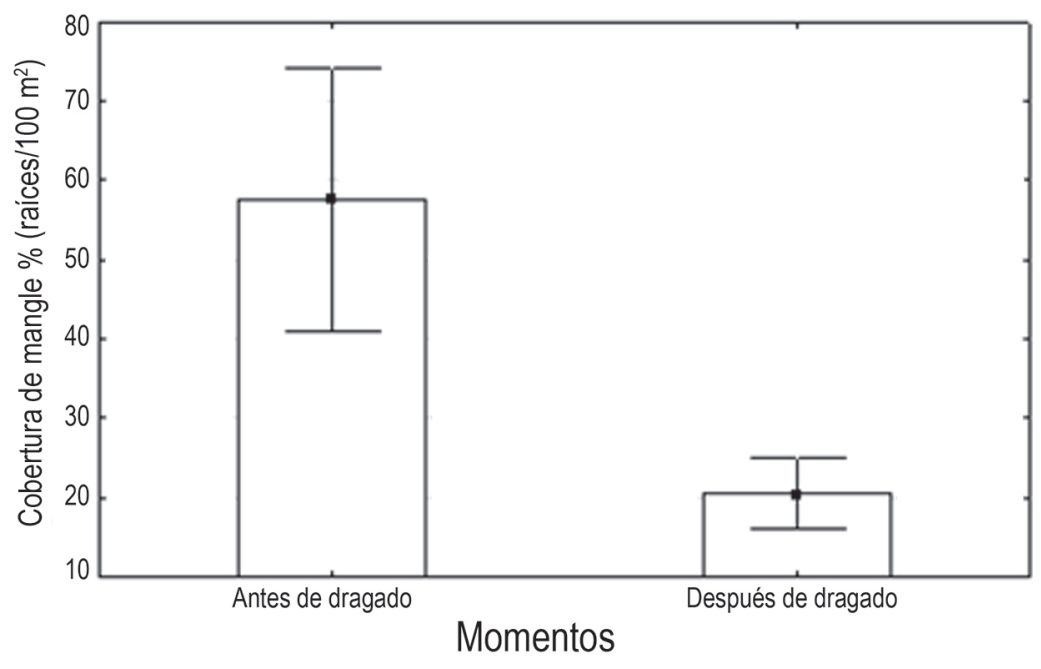

Media
I Media \pm Int.conf $95 \%$

Fig. 4. Cobertura de mangle en las orillas del canal antes y después del dragado. (Diferencias significativas, $P<0.001$, Prueba U de Mann-Whitney)

Fig. 4. Cover of mangrove at the channel sides, before and after the dredging. (Significant differences $P<0.001$, Mann-Whitney U Test)

sobre todo por la reducción de la cobertura de mangle rojo ( $R$. mangle) hacia las secciones finales y la casi desaparición del mangle prieto $(A$. germinans). De modo general, la cobertura total del manglar del canal se redujo en ambas secciones luego del dragado $(P<0.001$, Prueba $\mathrm{U}$ de Mann-Whitney) (Fig. 4). Esto se debe a que conjuntamente con el dragado de la laguna se realizaron acciones de limpieza en el canal de comunicación al mar de Laguna Larga, incluyendo el desbroce de vegetación perteneciente a las riberas del canal, fundamentalmente el mangle, con el objetivo de mejorar la circulación de agua hacia el interior de esta. En relación con la sombra, no se pudieron encontrar diferencias significativas antes y después del dragado $(P=0.1809$, Prueba U de MannWhitney). No obstante, parece tener una tendencia al incremento. Esto se explica por un incremento de la sombra producida por otras especies diferentes a los mangles evaluados, como son los casos del patabán (Laguncularia racemosa L.) y la llana (Conocarpus erecta L.), según Salvat-Torres et al. (2013). Al analizar la composición del fondo, en el centro del canal, se encontró que se incrementó significativamente el área cubierta de arena después del dragado $(P<0.001$, Prueba $U$ de Mann-Whitney) (Fig. 5) y se redujo el porcentaje de área ocupada por cascajos y troncos caídos $(P<0.001$, Prueba $\mathrm{U}$ de Mann-Whitney) (Fig. 6). El resto de las variables evaluadas (área cubierta de algas, T. testudinum y piedra) no presentaron diferencias significativas antes y después del dragado.

Al comparar estadísticamente los parámetros fisicoquímicos evaluados se 
encontraron diferencias significativas antes y después del proceso de dragado solo en la profundidad del canal $(P$ $<0.05$, Prueba U de Mann-Whitney)
(Fig. 7) y la salinidad $(P<0.01$, Prueba U de Mann-Whitney) (Fig. 3b). El resto de las variables evaluadas no mostraron diferencias significativas (Cuadro 2).

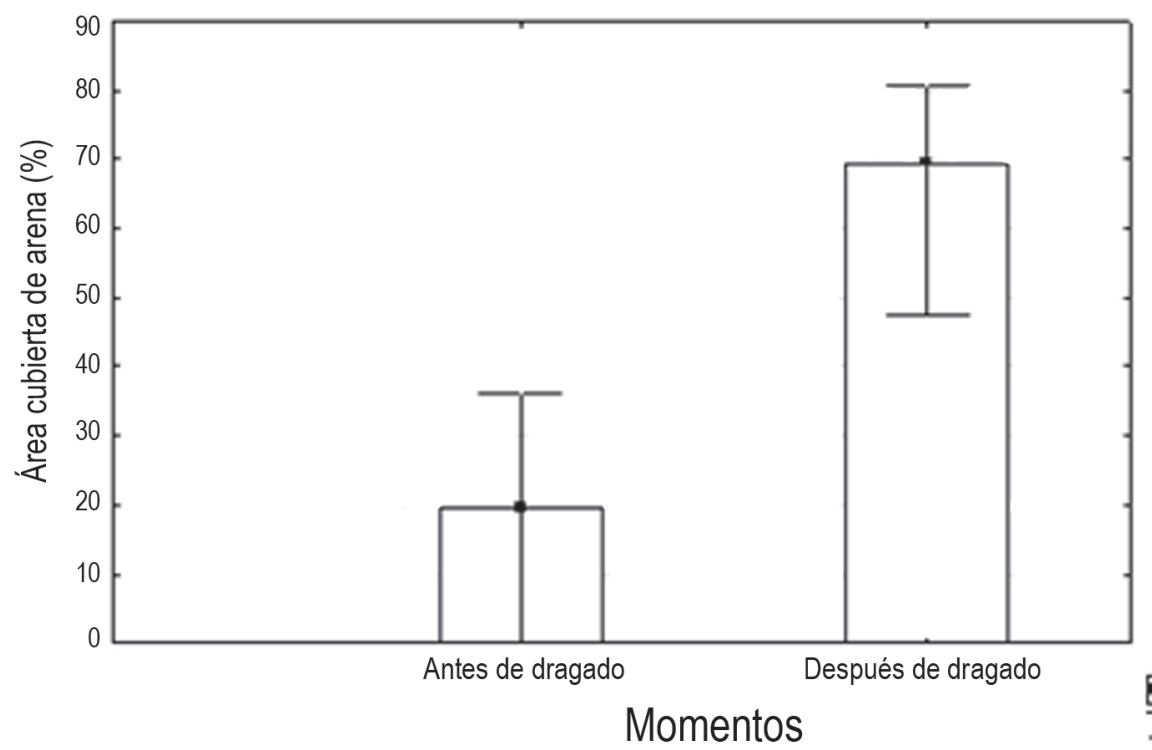

巳 Media
I Min.-máx.

Fig. 5. Área cubierta de arena en el centro del canal antes y después del dragado. (Diferencias significativas, $P<0.001$, Prueba U de Mann-Whitney)

Fig. 5. Area covered by sand in the middle of the channel before and after the dredging. (Significant differences, $P<0.001$, Mann-Whitney U Test)

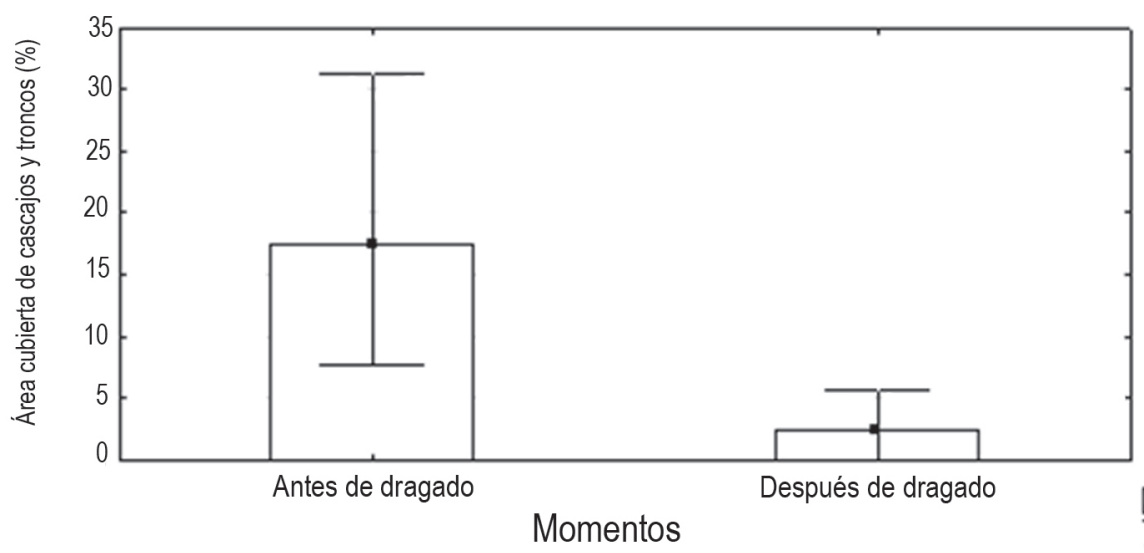

Media I Min.-máx

Fig. 6. Área cubierta de cascajos y troncos en el centro del canal antes y después del dragado. (Diferencias significativas, $P<0.001$, Prueba U de Mann-Whitney)

Fig. 6. Area covered by gravel and logs in the middle of the channel before and after the dredging. (Significant differences, $P<0.001$, Mann-Whitney U Test) 


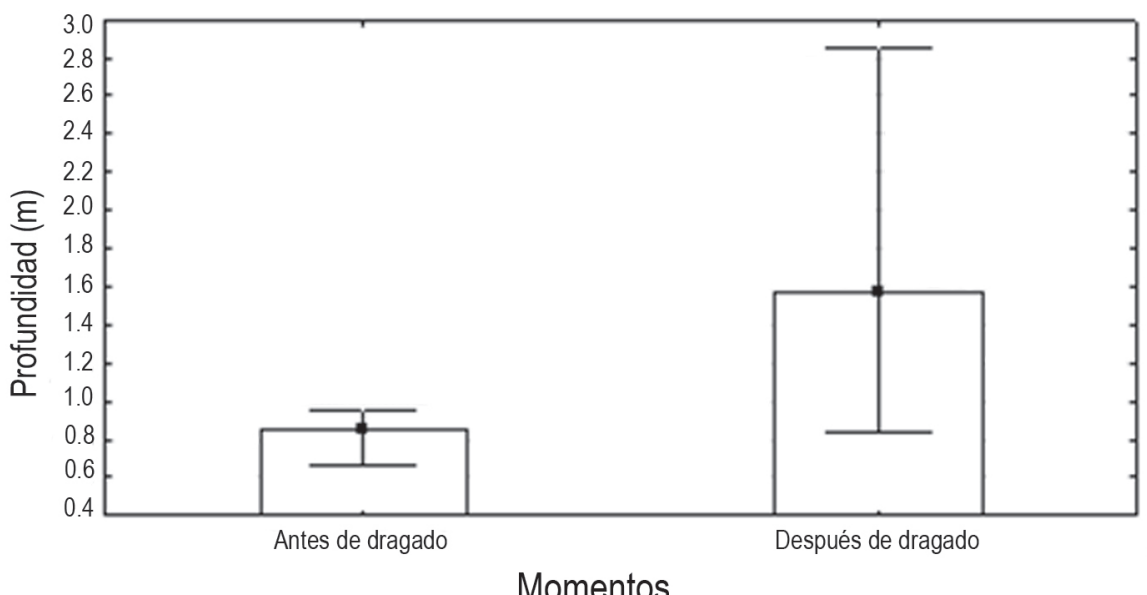

@ Media I Min.-máx

Fig. 7. Profundidad del canal de comunicación al mar de Laguna Larga antes y después del proceso de dragado. (Diferencias significativas, $P<0.05$, Prueba U de Mann-Whitney) Fig. 7. Depth of the Laguna Larga estuarine channel before and after the dredging process. (Significant differences, $P<0.05$, Mann-Whitney U Test)

De las variables $\mathrm{pH}$ y oxígeno disuelto no se tenían datos previos al dragado, pero al analizarse después del dragado, ambas no mostraron diferencias significativas. Además, fueron tabulados los datos de abundancia media total (individuos $/ 100 \quad \mathrm{~m}^{2}$ ) \pm error estándar, y frecuencia de observación, en los censos de las especies más conspicuas antes del dragado en el canal de comunicación al mar de Laguna Larga (Cuadro 3). También se tabularon los resultados del ANOVA de Friedman: densidad de peces en los diferentes años de estudio (individuos $/ 100 \mathrm{~m}^{2}$ ) (Cuadro 4) y la densidad media total (individuos/100 $\mathrm{m}^{2}$ ) antes y después del proceso de dragado. Análisis de ANOVA de Friedman: Wilcoxon Prueba de parejas sincronizadas (Cuadro 5).

\section{DISCUSIÓN}

El análisis de datos mostró que la densidad de peces disminuyó incluso a partir del 2008 y se mantuvo establemente reducida en los años 2014 y 2015. Concordantemente, los valores mínimos de densidad registrados se obtuvieron en la etapa posterior al dragado. Especies como A. saxatilis, $H$. bivittatus y $S$. leucostictus mostraron una disminución gradual de sus densidades (disminuyeron con el paso del tiempo), mientras que L. griseus lo hizo de forma más irregular (en el período del 2007-2008 aumentó y en el 20142015 disminuyó), pero igualmente con valores mínimos en la etapa posterior al dragado. La acción del hombre sobre este ecosistema dio lugar a la drástica disminución de la densidad de peces en el canal, lo cual concuerda con lo referido por Benedetti-Cecchi et al. (2001). 
Cuadro 2. Resumen de los parámetros fisicoquímicos en el canal de comunicación al mar de Laguna Larga luego del proceso de dragado

Table 2. Summary of physico-chemical parameters in the estuarine channel of Laguna Larga after the dredging process

\begin{tabular}{lcccccc}
\hline $\begin{array}{l}\text { Parámetros } \\
\text { fisicoquímicos }\end{array}$ & Media & $\begin{array}{l}\text { Intervalo de } \\
\text { confianza } \\
\mathbf{9 5} \%\end{array}$ & Mediana & Mínimo & Máximo & $\begin{array}{l}\text { Desviación } \\
\text { típica }\end{array}$ \\
\hline Profundidad (m) & 1.57 & $1.2-1.9$ & 1.10000 & 0.9 & 3.0 & 0.8 \\
Velocidad (m/s) & 0.36 & $0.34-0.38$ & 0.35714 & 0.25 & 0.47 & 0.05 \\
Temperatura ( $\left.{ }^{\circ} \mathbf{C}\right)$ & 25.1 & $24.2-26.0$ & 25.90000 & 21.0 & 28.0 & 2.2 \\
Salinidad (PSU) & 35.6 & $35.2-36.0$ & 35.65000 & 33.8 & 37.6 & 0.9 \\
$\mathbf{O}_{2}$ Disuelto (mI/l) & 5.3 & $5.15-5.51$ & 5.35000 & 4.45 & 6.25 & 0.43 \\
$\mathbf{p H}$ & 7.6 & $7.5-7.8$ & 7.55000 & 7.1 & 8.9 & 0.4 \\
\hline
\end{tabular}

Cuadro 3. Abundancia media total (individuos $/ 100 \mathrm{~m}^{2}$ ) \pm error estándar y frecuencia de observación, en los censos de las especies más conspicuas antes del dragado en el canal de comunicación al mar de Laguna Larga

Table 3. Total average abundance (individuals $/ 100 \mathrm{~m}^{2}$ ) \pm standard error, and frequency of observation in the surveys of the most conspicuous species before the dredging in the estuarine channel of Laguna Larga

\begin{tabular}{lll}
\hline Especies & $\begin{array}{l}\text { Abundancia Media } \\
\pm \mathrm{EE}\end{array}$ & $\begin{array}{l}\text { Frecuencia } \\
(\%)\end{array}$ \\
\hline Clupleidae & $83.96 \pm 15.99$ & 10 \\
Abudefduf saxatilis & $64.93 \pm 2.93$ & 87 \\
Halichoeres bivittatus & $13.82 \pm 0.59$ & 82 \\
Stegastes leucostictus & $11.76 \pm 0.52$ & 78 \\
Lutjanus griseus & $9.78 \pm 0.39$ & 95 \\
Scarus iserti-taenopterus & $7.43 \pm 0.46$ & 55 \\
Eucinostomus sp. & $6.22 \pm 0.32$ & 82 \\
Haemulon parra & $5.44 \pm 0.21$ & 61 \\
Gerres cinereus & $4.75 \pm 0.14$ & 94 \\
Lutjanus apodus & $3.29 \pm 0.13$ & 70 \\
Haemulon sciurus & $2.65 \pm 0.11$ & 77 \\
Sparisoma aurofrenatum & $2.62 \pm 0.13$ & 59 \\
Sphyraena barracuda & $1.93 \pm 0.21$ & 77 \\
Chaetodon capistratus & $1.24 \pm 0.06$ & 44 \\
Sphoeroides testudineus & $1.15 \pm 0.07$ & 49 \\
Haemulon flavolineatum & $1.03 \pm 0.13$ & 14 \\
Stegastes fuscus & $1.00 \pm 0.05$ & 48 \\
\hline
\end{tabular}


Cuadro 4. Análisis de ANOVA de Friedman en los diferentes años de estudio: Prueba de ANOVA de Friedman y Kendall Coeff. De Concordancia: ANOVA Chi Sqr. (N =6, $\mathrm{df}=3$ ) $=8.600000 P=0.03$ (Densidad de peces.stw)

Table 4. Analysis of Friedman's ANOVA for the different years of study: ANOVA test by Friedman and Kendall Coeff. From Concordance: ANOVA Chi Sqr. $(\mathrm{N}=6, \mathrm{df}=3)$ $=8.600000 P=0.03$ (Density of fish.stw)

\begin{tabular}{cccc}
\hline Años & N & Suma de Rangos & Media de Rangos \\
\hline 2007 & 216 & 133797.5 & 6194.329 \\
2008 & 336 & 201553.0 & 5998.601 \\
2014 & 176 & 51026.5 & 2899.233 \\
2015 & 48 & 10180.5 & 2120.938 \\
\hline
\end{tabular}

Cuadro 5. Densidad media total (individuos $/ 100 \mathrm{~m}^{2}$ ) antes y después del proceso de dragado. Análisis de ANOVA de Friedman: Wilcoxon Prueba de parejas sincronizadas. (Diferencias significativas, $P<0.05$ ) Table 5. Total average density (individuals $/ 100 \mathrm{~m}^{2}$ ) before and after the dredging process. Analysis of Friedman's ANOVA: Wilcoxon Test of synchronized pairs. (Significant differences, $P<0.05$ )

\begin{tabular}{llll}
\hline $\mathbf{N}$ & $\mathbf{T}$ & $\mathbf{Z}$ & $\mathbf{p}$ \\
\hline 28 & 3900.000 & 3734.513 & 0.000188 \\
\hline
\end{tabular}

La densidad media de las especies más comunes, antes y después del proceso de dragado, mostró el mismo comportamiento. El número de individuos en el canal disminuyó sustancialmente después de que se realizó el dragado en Laguna Larga. Contribuye a esta conclusión la perspectiva individual de la variación espacial y temporal que han sufrido estas especies después del dragado. La abundancia de $A$. saxatilis, $H$. bivittatus, L. griseus y S. leucostictus disminuyó y algunas especies como:
L. griseus, H. sciurus y $H$. parra se reubicaron en otras zonas del canal (sección IB más cercana al mar, de FB más cercana a la laguna). Sin embargo, especies de tamaño pequeño como: $A$. saxatilis, $H$. bivittatus y $S$. leucostictus se mantuvieron en las secciones iniciales debido, posiblemente, al predominio de los refugios pequeños en dichas secciones (Sierra et al. 2001; Anticamara et al. 2010). En el caso particular de $S$. leucostictus, la disponibilidad de refugios ubicados en el fondo, como algunos troncos secos y escombros, pudo incidir en que esta especie prefiera estar sobre todo en esa zona (secciones IA-IB). S. leucostictus es fuertemente territorial y depende en gran medida de un refugio adecuado para su presencia, como exponen Valdés-Muñoz \& Mochek (2001). Esta variación espacial parece estar relacionada con las características de hábitat, lo cual concuerda con Pombo et al. (2005), quienes plantean que la baja segregación espacial de peces en lo referente a preferencia alimenticia indica que, en una laguna y su canal 
adyacente con amplia disponibilidad de alimentos, los factores abióticos parecen tener un mayor efecto en la distribución de los peces que las interacciones biológicas.

La zona inicial del canal brinda un mejor refugio para peces pequeños, siendo estos a su vez los más abundantes en todo el canal, pero especialmente en la zona inicial. Las secciones iniciales están dominadas por las raíces comunes de mangle prieto, las cuales brindan un mejor refugio a especies pequeñas. Por otra parte, hacia el final del canal hay una mejor representación de las raíces de mangle rojo, que presentan entre sí grandes espacios y mayor área de sombra, proveyendo refugio a especies de peces de mayor tamaño que las encontradas en la sección inicial.

Estos aspectos han sido documentados en trabajos como los realizados por Mochek \& ValdésMuñoz (1983); Dahlgren \& Eggleston (2000); Valdés-Muñoz \& Mochek (2001), donde concluyen que la disponibilidad de refugio, alimento y la depredación son factores críticos para la distribución de las especies. Estos resultados también apoyan a otros autores (Stunz et al. 2002; Cocheret de la Morinière et al. 2004), que han planteado que la abundancia de los peces en los manglares es específica para cada especie y está influenciada por su comportamiento y por las características del hábitat.

Es de destacar que las mayores densidades y diferencias en la variación temporal se notaron, principal- mente, en la zona inicial del canal y no al final, resultado que indica la posible conexión entre el mar y esa zona del canal. Esto concuerda con lo planteado por García-Cagide et al. (2001) y Salvat-Torres et al. (2010; 2013), quienes mencionan que las poblaciones de peces, del canal de comunicación al mar de Laguna Larga, muestran variaciones en el tiempo, al parecer relacionadas con la movilidad intrínseca de los peces, migraciones reproductivas, reclutamiento y mortalidad diferencial asociada a la depredación.

Estas diferencias en las variaciones en el tiempo y las mayores densidades encontradas en las zonas iniciales del canal y no al final podrían estar dadas porque, luego del dragado, los peces presentes en su canal de comunicación se hayan desplazado hacia el interior de la laguna buscando menos disturbios antrópicos, así como mayor disponibilidad de refugio entre las raíces del mangle rojo que bordean toda la laguna, corroborado por SalvatTorres et al. (2013).

Los resultados obtenidos mostraron segregación espacial de la abundancia de las especies en la comunidad de peces presentes en el canal. La distribución se mantiene similar a la descrita por Salvat-Torres et al. (2010). La variación espacial de la abundancia de los peces en el canal de comunicación al mar de Laguna Larga está estrechamente relacionada con las características del hábitat, por lo tanto si estas cambian, también debe cambiar el patrón de distribución. Este comportamiento que presentan los peces que 
viven en los manglares se explica de igual manera en otros estudios como los realizados por Cocheret de la Morinière et al. $(2003 ; 2004)$, quienes plantean que la densidad y el tamaño de los peces que habitan en los manglares están fielmente relacionados con el refugio. Algunos estudios experimentales han demostrado que el incremento en la complejidad estructural de los manglares reduce la eficiencia de la depredación (Primavera, 1997; Laegdsgaard \& Johnson, 2001), por lo que la depredación es una variable que podría estar determinando la distribución de los peces en el canal de Laguna Larga, debido al cambio en la complejidad estructural, la cual disminuyó.

El hecho de que las mayores abundancias de peces se encontraran en las zonas iniciales del canal (zonas más cercanas al mar: IA-IB), especialmente en la sección IB, se debe a que en este tramo del canal las características del hábitat, tanto del fondo como sus riberas, no han cambiado mucho durante el tiempo. No siendo así para el resto de las secciones del canal, en las cuales se realizaron algunos procesos de tala de tramos de mangles, recogidas de ramas y troncos de vegetación que forman estructuras de refugios para peces, planteamiento que concuerda con Villéger et al. (2010); Mohamed-Harris \& Vinobaba (2013), quienes sugieren que la diversidad de la comunidad de peces disminuye por un empobrecimiento de la vegetación del fondo. Estas acciones fueron realizadas para mejorar la circulación de agua hacia el interior de la laguna y así mejorar la calidad de esta. Sin embargo, influyó en las características del hábitat del canal, reduciendo sustancialmente la disponibilidad de refugios para la mayoría de los peces que dependía de ellos, como L. apodus, L. griseus, H. sciurus, entre otras especies. Los refugios que quedaron no tenían el suficiente espacio para especies como L. apodus, L. griseus, H. sciurus, sobre todo en sus estadios adultos, pero sí para especies pequeñas como los píntanos (A. saxatilis) y la chopita de lomo violeta ( $S$. leucostictus), que utilizan espacios limitados para refugiarse, como son los casos de las raíces del mangle prieto ( $A$. germinans) y troncos caídos que se encuentran en esta zona del canal según Salvat-Torres et al. (2013). Lo antes planteado concuerda con Caballero-Vázquez \& Vega-Cendejas (2012), una alta complejidad del hábitat provee mayor cantidad de alimentos y refugio contra depredadores.

El análisis individual de la densidad media de las especies dominantes en el canal mostró diferentes patrones, posiblemente debido a la mayor disponibilidad de refugio en esta sección, el cambio de nicho de L. griseus puede estar dado por la presencia de refugio acorde al tamaño de esta especie, el cual varió entre $5-10 \mathrm{~cm}$ de longitud y en ocasiones esporádicas presentó un tamaño de $15 \mathrm{~cm}$ según Salvat-Torres et al. (2013).

El patrón de distribución de las especies más conspicuas fue similar al descrito por Salvat-Torres et al. 
(2010), excepto para L. griseus, el cual cambió sus preferencias de nicho, desde el final de los canales FA-FB, hacia la sección IB. Todo esto está muy influenciado por el comportamiento de los peces, coincidiendo con lo sugerido por Mesa et al. (2010), cada especie percibe el ambiente en un rango de escala único y esto responde a la variabilidad individual.

Por ejemplo, Mochek \& ValdésMuñoz (1983) encontraron que a diferencia del arrecife coralino, las especies de peces como L. griseus, L. apodus, $H$. sciurus y $H$. parra mostraban durante el día un comportamiento de reacción defensiva, lo que explica la atracción que muestran estos peces hacia el manglar como refugio, en horas del día.

El canal no era homogéneo en cuanto a las características de sus riberas antes del proceso de dragado, sus bordes tenían más cobertura de raíces y sombra de mangle rojo hacia el final del canal, mientras que en su inicio, predominaban los neumatóforos y raíces comunes de mangle prieto. Con respecto al fondo del canal, el predominio de algas macroscópicas es mayor en el inicio del canal y los hidrozoos y Thalassia predominan hacia el final de este.

El incremento de arena se explica por el aumento del intercambio entre el agua de mar y el agua del canal, después que aumentó el flujo de esta ocasionado por el dragado de la laguna, cuya circulación influye en el canal. La reducción de los cascajos y troncos caídos en el centro del canal está relacionados con las acciones de limpieza realizadas en este, con el objetivo de mejorar la circulación de agua hacia el interior de la laguna. El cambio en las características del hábitat influyó en la comunidad de peces, debido a que las características del sedimento, la heterogeneidad del sustrato y la cobertura vegetal también afectan la distribución de peces, dada su influencia en la disponibilidad de presas (Mesa et al. 2010) y la protección contra depredadores (Pombo et al. 2005).

\section{Al comparar estadísticamente} los parámetros fisicoquímicos evaluados se encontraron diferencias significativas antes y después del proceso de dragado solo en la profundidad del canal $(P<0.05$, Prueba U de Mann-Whitney) (Fig. 7) y la salinidad $(P<0.01$, Prueba $U$ de Mann-Whitney) (Fig. 3b), antes del dragado los valores oscilaron entre 36.7-37.1 PSU según HernándezRoque (2007), y después entre 34.636.6 PSU. Lo anterior indica que a pesar de que no hubo variaciones significativas la salinidad se redujo, lo cual podría estar dado por el aumento del intercambio entre el agua de mar y el agua del canal después de que aumentó el flujo ocasionado por el dragado de la laguna, cuya circulación influye en el canal, pero no se considera el cambio de salinidad como la causa de la reducción de las poblaciones de peces, ya que los peces pueden aceptar un rango de salinidad de 0 a 55 PSU, según lo establecido por Ayala-Pérez et al. (2003). A 
pesar de ello se resalta la importancia de la salinidad para este estudio, debido a que los fuertes gradientes direccionales de salinidad, materia orgánica, nutrientes y concentración de oxígeno presentes en los canales que comunican las lagunas costeras actúan como filtro en la colonización de especies (Garrido et al. 2011).

\section{CONCLUSIONES}

El proceso de restauración ambiental de Laguna Larga produjo una disminución de la complejidad física del hábitat solo en el canal de entrada a la laguna, reflejada en la disponibilidad de refugios y consecuentemente determinó la disminución de la densidad de especies encontradas en su canal de comunicación al mar. Las especies dominantes antes y después del proceso de dragado fueron las mismas, pero con variaciones espaciales particulares como es el caso de L. griseus, la cual cambió sus preferencias de nicho desde las secciones finales del canal hasta la sección inicial.

\section{BIBLIOGRAFÍA}

Anticamara, J. A., Zeller, D. A. \& Vicent, A. C. J. (2010). Spatial and temporal variation of abundance, biomass and diversity within marine reserves in the Philippines. Divers. Distrib., 16(4), 529536. http://dx.doi.org/10.1111/ j.1472-4642.2010.00661.x Ayala-Pérez, L. A., Ramos, J. M. \& Flores, D. H. (2003). La comunidad de peces de la Laguna de Términos: estructura actual comparada. Rev. Biol. Trop., 51(3-4), 783-793.

Benedetti-Cecchi, L., Rindi, F., Bertocci, I., Bulleri, F. \& Cinelli, F. (2001). Spatial variation in development of epibenthic assemblages in a coastal lagoon. Estuar. Coast. Shelf Sci., 52, 659-668. http:// dx.doi.org/10.1006/ecss.2001.0775 Brock, V. E. (1954). A preliminary report on a method of estimating reef fish populations. J. Wildl. Manage., 18(3), 297-308. http://dx.doi. org/10.2307/3797016

Caballero-Vázquez, J. A. \& VegaCendejas, M. E. (2012). Spatial patterns of diversity at local and regional scales in a tropical lagoon. Neotrop. Ichthyol., 10, 99108. http://dx.doi.org/10.1590/ S1679-62252012000100010

Clarke, K. R. \& Gorley, R. N. (2006). User manual/tutorial. Plymouth, England: PRIMER-E Ltd.

Claro, R. \& Parenti, L. R. (2001). The marine ichthyofauna of Cuba. In R. Claro, K. C. Lindeman \& L. R. Parenti (Eds.), Ecology of the marine fishes of Cuba (pp. 21-32). Washington \& London, EE.UU. \& England: Smithsonian Institution Press.

Cocheret de la Morinière, E., Pollux, B. J. A., Nagelkerken, I. \& van der Velde, G. (2003). Diet shifts of Caribbean grunts (Haemulidae) and snappers (Lutjanidae) and the relation with nursery to coral reef migrations. Estuar. 
Coast. Shelf Sci., 57(5-6), 10791089. http://dx.doi.org/10.1016/ S0272-7714(03)00011-8

Cocheret de la Morinière, E., Nagelkerken, I., van der Meij, H. \& van der Velde, G. (2004). What attracts juvenile coral reef fish to mangrove: habitat complexity or shade? Mar. Biol., 144, 139-145. http://dx.doi. org/10.1007/s00227-003-1167-8

Contreras, F. \& Castañeda, O. (2004). La biodiversidad de las lagunas costeras. Rev. Ciencias, 76, 46-56.

Dahlgren, C. P. \& Eggleston, D. B. (2000). Ecological processes underlying ontogenetic habitat shifts in a coral reef fish. Ecol., 81(8), 2227-2240. http:// dx.doi org/10.1890/00129658(2000)081[2227:EPUOHS] 2.0.CO;2

Farré, M., Lombarte, A., Recasens, L., Maynou, F. \& Tuset, V. M. (2015). Habitat influence in the morphological diversity of coastal fish assemblages. J. Sea Res., 99, 107117. http://dx.doi.org/10.1016/j. seares.2015.03.002

García-Cagide, A. R., Claro, R. \& Koshelev, B. V. (2001). Reproductive patterns of fishes of the Cuba Shelf. In R. Claro, K. C. Lindeman \& L. R. Parenti (Eds.), Ecology of the Marine Fishes of Cuba (pp. 73-114). Washington \& London, EE.UU. \& England: Smithson an Institution Press.

Garrido, J., Pérez-Bilbao, A. \& Benetti, C. J. (2011). Biodiversity and Conservation of Coastal
Lagoons. In O. Grillo \& G. Varona (Eds.), Ecosystems Biodiversity (pp. 1-28). Vigo, España: InTech, Universidad de Vigo. http://dx.doi. org/10.5772/24934

González-De Zayas, R. (2012). Balance de nitrógeno y fósforo en una laguna costera tropical (Laguna Larga, Cuba). Tesis de doctorado no publicada, Universidad Nacional Autónoma de México, México.

González-Sansón, G. \& Aguilar, C. (2003). Variaciones espaciales y temporales en la abundancia de las especies dominantes de peces de arrecife de coral en la costa de Ciudad de La Habana, Cuba. Rev. Invest. Mar, 24(2), 99-110.

Guimarais-Bermejo, M. (2007). Ecología de Ruppia maritima L. en Laguna Larga, Cayo Coco, Cuba. Tesis de maestría no publicada, Universidad de La Habana, Cuba.

Hernández-Roque, L. (2007). Calidad de agua y gestión ambiental de Laguna Larga, Cayo Coco, Cuba. Tesis de maestría no publicada, Universidad de Matanzas Camilo Cienfuegos, Cuba.

Knowlton, N. (2001) The future of coral reefs. Proc. Natl. Acad. Sci., 98, 5419-5425. http://dx.doi. org/10.1073/pnas.091092998

Laegdsgaard, P. \& Johnson, C. (2001). Why do juvenile fish utilize mangrove habitats? $J$. Exp. Mar. Biol. Ecol., 257, 229253. http://dx.doi.org/10.1016/ S0022-0981(00)00331-2 
Locatelli, E. \& Petersen, R. G. (1975). Algunos trucos útiles en estadísti$c a$. Oregon, EE.UU.: Corvallis, International Plant Protection Center. Menéndez, L. \& Priego, A. (1994). Los manglares de Cuba: Ecología. En E. D. Suman (Ed.), El ecosistema de manglar en América Latina y la Cuenca del Caribe, su manejo y conservación (pp. 64-75). La Habana, Cuba: Science \& The Tinker Foundation.

Mesa, G. L., Molinari, A., Gambaccini, S. \& Tunesi, L. (2010). Spatial pattern of coastal fish assemblages in different habitats in Noth-Western Mediterranean. Mar. Ecol., 32, 104-114. http://dx.doi.org/10.1111/ j.1439-0485.2010.00404.x

Mochek, A. D. \& Valdés-Muñoz, E. (1983). Acerca de la conducta de los peces de la comunidad costera en la plataforma cubana. Rev. Cienc. Biol., 9, 87-106.

Mohamed-Harris, J. \& Vinobaba, P. (2013). Assessment the Present Status of Batticaloa Lagoon, Sri Lanka by means of Water Quality, Fish Diversity Indices and Pollution Indicating Planktons. J. Biodivers. Endangered Spec., 2(1), 2-6. http:// dx.doi.org/10.4172/.1000105

Mumby, P. J., Edwards, A. J., Arias-González, J. E., Lindeman, K. C., Blackwell, P. G., Gall, A., Gorczynsha, M. I. \& Llewellyn, G. (2004). Mangroves enhance the biomass of coral reef fish communities in the Caribbean. Nature, 427(6974), 533-536. http://dx.doi. org/10.1038/nature02286
Nagelkerken, I., Dorenbosch, M., Verberk, W. C. E. P., Cocheret de la Morinière, E. \& van der Velde, G. (2000). Importance of shallow-water biotopes of a Caribbean bay for juvenile coral reef fishes: patterns in biotope association, community structure and spatial distribution. Mar. Ecol. Prog. Ser., 202, 175-192. http://dx.doi. org/10.3354/meps202175

Nagelkerken, I., Kleijnen, S., Klop, T., van den Brand, R. A. C. J., Cocheret de la Morinière, E. \& van der Velde, G. (2001). Dependence of Caribbean reef fishes on mangroves and seagrass beds as nursery habitat: a comparison of fish fauna between bays with and without mangrove/seagrass beds. Mar. Ecol. Prog. Ser., 214, 225-235. http:// dx.doi.org/10.3354/meps214225

Nagelkerken, I., Roberts, C. M., van der Velde, G., Dorenbosch, M., van Riel, M. C., Cocheret de la Morinière, E. \& Nienhuis, P. H. (2002). How important are mangroves and seagrass beds for coral-reef fish? The nursery hypothesis tested on an island scale. Mar. Ecol., 244, 299-305. http:// dx.doi.org/10.3354/meps244299

Nixon, S. W. \& Fulweiler, R. W. (2009). Nutrient pollution, eutrophication, and the degradation of coastal marine ecosystems. In C. M. Duarte (Ed.), Global loss of coastal habitats: rates, causes and consequences (pp. 87-120). Bilbao, España: Fundación BBVA.

Olivera-Espinosa, Y. (2013). Evaluación de la superposición de nichos en los 
ensambles de moluscos marinos de una laguna costera en Cayo Coco, Cuba. Tesis de maestría no publicada, Universidad de La Habana, Cuba. Pombo, L., Elliott, M. \& Rebelo, J. E. (2005). Environmental influences on fish assemblage distribution of an estuarine coastal lagoon, Ria de Aveiro (Portugal). Sci. Mar., 69, 143-159. http://dx.doi.org/10.3989/ scimar.2005.69n1143

Primavera, J. H. (1997). Fish predation on mangrove-associated penaeids: The role of structures and substrate. J. Exp. Mar. Biol. Ecol., 215(2), 205-216. http://dx.doi.org/10.1016/ S0022-0981(97)00046-4

Rogers, C. S., Garrison, G., Grober, R., Hillis, Z. M. \& Franke, M. A. (1994). Coral Reef Monitoring Manual for the Caribbean and Western Atlantic. Virgin Islands National Park, EE. UU.: s.e.

Salvat-Torres, H. M., Pina-Amargós, F. \& González-Sansón, G. (2010). Variación espacial de la comunidad de peces asociados a un canal de mangles en Cayo Coco, Cuba. Rev. Invest. Mar., 31(2), 131-140.

Salvat-Torres, H. M., Pina-Amargós, F., Salvat-Quesada, M. \& González-Sansón, G. (2013). Variación temporal de la comunidad de peces en un canal de comunicación al mar en Cayo Coco, Cuba. Rev. Invest. Mar., 33(1), 1-13.

Sierra, L. M., Claro, R. \& Popova, O. A. (2001). Trophic biology of the marine fishes of Cuba. In R. Claro, K. C. Lindeman \& L. R. Parenti (Eds.), Ecology of the marine Fishes of Cuba (pp. 21-32). Washington \&
London, EE.UU. \& England: Smithsonian Institution Press.

Sirot, C., Villéger, S., Mouillot, D., Darnaude, A. M., Ramos-Miranda, J., Flores Hernández, D. \& Panfili, J. (2015). Combinations of biological attributes predict temporal dynamics of fish species in response to environmental changes. Ecol. Indic., 48, 147-156. http://dx.doi. org/10.1016/j.ecolind.2014.07.038

StatSoft, Inc. (2011). Statistica for Windows (Computer Program Manual), version 10.0 www.statsoft. com. Tulsa, EEUU.: Statsoft, Inc.

Stunz, G. W., Minello, T. J. \& Levin, P. S. (2002). Growth of newly settled red drum Sciaenops ocellatus in different estuarine habitat types. Mar Ecol.Prog. Ser., 238, 227-236. http:// dx.doi.org/10.3354/meps238227

Valdés-Muñoz, E. \& Mochek, A. D. (2001). Behavior of marine fishes of the Cuban: Ecology of the marine fishes of Cuba. In R. Claro, K. C. Lindeman \& L. R. Parenti (Eds.), Behavior of marine fishes of the Cuban (pp. 5872). Washington \& London, EE.UU. \& England: Smithsonian Institution Press. Villéger, S., Miranda, J. R., Flores-Hernández, D. \& Mouillot, D. (2010). Contrasting changes in taxonomic vs. functional diversity of tropical fish communities after habitat degradation. Ecol. Appl., 20, 1512-1522. http://dx.doi.org/10.1890/09-1310.1.

Zar, J. H. (1996). Biostatistical analysis (3ra. Ed.). New Jersey, EE.UU.: Prentice Hall.

Zúñiga, A. \& González, D. (2000). Las causas de los procesos erosivos de las playas del Polo turístico Jardines del Rey. Enlace, 7(34), 25-29. 